\title{
MEMORY FOR SINGLE ITEMS, WORD PAIRS, AND TEMPORAL ORDER OF DIFFERENT KINDS IN A PATIENT WITH SELECTIVE HIPPOCAMPAL LESIONS
}

\author{
A.R. Mayes, C.L. Isaac, J.S. Holdstock, N.M. Hunkin, and D. Montaldi \\ University of Sheffield, Royal Hallamshire Hospital, Sheffield, U.K.
}

\author{
J.J. Downes \\ University of Liverpool, U.K. \\ C. MacDonald \\ University of Manchester, U.K.
}

\author{
E. Cezayirli and J.N. Roberts \\ Magnetic Resonance and Image Analysis Research Centre, University of Liverpool, U.K.
}

\begin{abstract}
One kind of between-list and two kinds of within-list temporal order memory were examined in a patient with selective bilateral hippocampal lesions. This damage disrupted memory for all three kinds of temporal order memory, but left item and word pair recognition relatively intact. These findings are inconsistent with claims that (1) hippocampal lesions, like those of the medial temporal lobe (MTL) cortex, disrupt item and word pair recognition, and that (2) hippocampal lesions disrupt temporal order memory and item recognition to the same degree. Not only was word pair recognition intact in the patient, but further evidence indicates that her recognition of other associations between items of the same kind is also spared so retrieval of such associations cannot be sufficient to support within-list temporal order recognition. Rather, as other evidence indicates that the patient is impaired at recognition of associations between different kinds of information, within-list (and possibly between-list) temporal order memory may be impaired by hippocampal lesions because it critically depends on retrieving associations between different kinds of information.
\end{abstract}

\section{INTRODUCTION}

Global amnesia is a disorder of pre- and post-morbid recognition and recall memory. Memory is impaired for both factual and episodic information that is encountered post-morbidly. It is also impaired for pre-morbidly encountered facts and episodes except when information has been greatly overlearnt pre-morbidly, as applies most typically to factual information. This condition is caused by medial temporal lobe (MTL), midline diencephalic, basal forebrain, and possibly other lesions (see Mayes, 1988). Memory for the temporal order of presented items has usually been studied in such patients in order to determine whether their memory for this kind of contextual information is more impaired than their recognition memory for individual items (see Mayes, 1988). Studies have

Requests for reprints should be addressed to A.R. Mayes, Department of Psychology, University of Liverpool, PO Box 147, Liverpool, L69 3BX, UK. Tel: +44 (0)151 794 2957; Fax: +44 (0)151 7942945.

This work was supported by a Medical Research Council programme grant (G9300193) awarded to A.R. Mayes. 
often included patients with Korsakoff's syndrome, who typically have damage that includes midline diencephalic structures (see Victor, Adams, \& Collins, 1989), as well as patients with damage to MTL structures, in order to examine the possibility that the pattern of temporal order memory impairment differs across these two patient groups. In such studies, recognition of single items is usually matched between patient and control groups by giving the patients more time to study the items or by testing them at shorter delays than those used with control subjects. Memory for the temporal order of the items is then investigated under the same conditions. If it is impaired, it can be concluded that temporal order memory is more impaired than item recognition in amnesics provided that the matching manipulation affects normal subjects' temporal order memory and item recognition to a similar degree.

Three kinds of temporal order memory paradigm have been used with amnesic patients. These paradigms include: (1) tests of recency (e.g., Milner, 1971), in which subjects must decide which of two items previously presented in a single list was seen most recently; (2) tests of list discrimination (e.g., Squire, Nadel, \& Slater, 1981; Kopelman, 1989), in which subjects must decide in which of two sequentially presented lists a recognised item was presented; and (3) tests of temporal sequencing (e.g., Mangels, 1997; Shimamura, Janowsky, \& Squire, 1990), in which subjects must reconstruct from memory the presentation order of a list of items.

Whether these tests tap the same or slightly different memory processes has never been systematically investigated. Two sources of evidence suggest, however, that tests of recency depend on different processes from tests of list discrimination and temporal sequencing. First, dissociations between temporal sequencing and recency test performance have been reported in normal subjects. For example, Greene, Thapar, and Westerman (1998) found that generating words as opposed to reading them led to worse temporal sequencing memory, but did not affect recency test performance. Second, similar dissociations between performance on tests of recency and performance on tests of list discrimina- tion and temporal sequencing have been found in studies of amnesia. Thus, although, as discussed later, the results of studies testing these latter two forms of temporal memory are not in complete agreement with each other, patients with global amnesia have always been impaired on such tests. In contrast, studies using tests of recency have typically found that amnesics were completely unimpaired. Thus, Milner, Corsi, and Leonard (1991) reported normal recency judgements in patients with unilateral temporal lobe lesions who showed impaired item recognition. In addition, Sagar, Gabrieli, Sullivan, and Corkin (1990) found that the densely amnesic patient HM showed normal recency judgements despite his recognition performance being little above chance. In unpublished work, we have replicated the results of these studies with a group of global amnesics that included patients with MTL damage as well as patients with midline diencephalic damage (Korsakoff patients).

Why these different subgroups of global amnesics seem to show relatively preserved memory on this kind of temporal order memory test is currently unknown. One explanation is that recency tests tap memory for a different form of temporal information from list discrimination and temporal sequencing tests, and that neither large MTL nor midline diencephalic lesions disrupt explicit memory for this kind of information. This seems very unlikely as an explanation of preserved memory, however, because global amnesics are impaired at explicit memory for all other forms of fact and event information including those forms of temporal information tapped by list discrimination and temporal sequencing tests.

An alternative explanation of why global amnesics have performed normally on tests of recency is that performance on such tests does not involve retrieving temporal information at all, but is based solely on judging the relative familiarity of the two list items being tested. Evidence that even item familiarity is not normal in global amnesics (Yonelinas, Kroll, Dobbins, Lazzara, \& Knight, 1998) suggests, however, that these patients should be impaired at recency tests if performance on them is based on judgements of relative familiarity. It nevertheless remains possible that, at least under 
certain circumstances, having impaired familiarity leaves recency test performance intact or may even facilitate it. Such facilitation could well occur if the older of two presented test items has been completely forgotten so that correct performance merely requires identifying that the other item's familiarity is above chance rather than judging which of the two items has higher familiarity. This possibility can be systematically investigated because although it predicts that amnesics' performance will be facilitated when the later, but not the earlier, item has been very recently presented, it also predicts that performance will be impaired when both items have been presented sufficiently long ago for their familiarity to be at chance. If the possibility is a correct account of how recency memory performance is mediated, then tests of recency memory cannot be regarded as true tests of temporal memory.

Although studies have consistently shown that amnesics are impaired at list discrimination and temporal sequencing tests, performance on these tests has not always been found to be disproportionately impaired relative to item recognition in patients with MTL damage. Kopelman (1989) found that both global amnesics with Korsakoff's syndrome, and those with Alzheimer's disease, who have damage in the MTLs, were disproportionately impaired on a list discrimination task. A similar disproportionate deficit in list discrimination performance was found in Korsakoff's syndrome patients by Squire (1982). However, Squire et al. (1981) found that patients who had undergone electroconvulsive therapy who, they argued, had suffered a functional disturbance of MTL structures, showed a level of list discrimination performance that was similar to that of the controls to whom they had been matched for item recognition. Shimamura et al., (1990) found a similar pattern of results in which Korsakoff patients showed greater impairments in temporal sequencing memory than item recognition, whereas amnesics with primarily MTL damage showed equivalent impairments. This investigation also found that patients with frontal lobe damage were impaired at the temporal sequencing task despite showing normal item recognition memory. On the basis of these results, the authors attributed the disproportionate temporal sequencing deficit in the Korsakoff group to the presence of incidental frontal lobe damage which, they argued, superimposed an additional and selective temporal order memory deficit onto the global amnesia caused by midline diencephalic damage. On this view, neither MTL nor midline diencephalic lesions alone should disrupt temporal order memory more than item recognition.

Other evidence suggests that disproportionate amnesic deficits in temporal order memory do not require additional frontal lobe dysfunction, however. For example, in the study by Kopelman (1989) described earlier, there was no evidence that the list discrimination deficit in either Korsakoff or Alzheimer groups was related to this kind of damage. Rather, correlational evidence suggested that it was related to the severity of the memory impairment, but not to frontal executive function impairments, in these patients. Kopelman, Stanhope, and Kingsley (1997) have found a similar pattern of correlations in global amnesics with large MTL lesions. In addition, these patients showed no evidence of reduced blood flow to the frontal lobes. Several further studies have also supported the contention that disproportionate deficits in list discrimination memory seen in patients with Korsakoff's syndrome need not be related to frontal lobe dysfunction (Hunkin \& Parkin, 1993; Hunkin, Parkin, \& Longmore, 1994; Parkin \& Leng, 1993). For example, Hunkin, Parkin, and Longmore (1994) found that although amnesics with Korsakoff's syndrome and those with MTL damage were similarly impaired in their recognition memory for target items, the Korsakoff amnesics were more impaired than the MTL patients on a test of list discrimination. This Korsakoff-specific disproportionate impairment did not correlate with performance on tests sensitive to frontal lobe dysfunction in these patients. Furthermore, in a previous case study, Parkin and Hunkin (1993) tested a patient with a hypothalamic tumour who showed no evidence of impairment on tests of executive function. Using the list discrimination procedure, they found that even though his item recognition performance was normal, he was impaired at list discrimination. 
These findings by Parkin and his colleagues of disproportionately impaired temporal order memory in Korsakoff, but not in MTL, amnesics have been used to support the hypothesis that amnesia may be functionally and anatomically heterogeneous in a specific kind of way. Thus, Parkin and colleagues (see Hunkin and Parkin, 1993; Parkin, 1992; Parkin and Hunkin, 1997; Parkin, Leng, \& Hunkin, 1990) have postulated that patients with damage to midline diencephalic structures suffer a different memory deficit from those with damage to MTL structures. They argue that this occurs, although the two regions are interconnected by the circuit of Papez, because the regions receive some different inputs, make some different outputs, and engage in somewhat different kinds of processing. Their hypothesis is based on the proposal that retrieval of contextual information, defined as spatiotemporal and other information that allows memories for events to be differentiated, plays a role in the recall/recognition of target (or attended) information and that encoding of contextual information into memory is disrupted by the kind of damage to diencephalic structures that is found in Korsakoff patients (Parkin, 1992; see Mayes, Meudell, \& Pickering, 1985). In contrast, the role of the MTL is postulated to be that of consolidating target information, context information, and presumably the associations between them, into memory. Patients with MTL damage are, therefore, hypothesised to be equally impaired at consolidating target and temporal as well as other kinds of contextual and associative information into memory according to the hypothesis of Parkin and his associates. MTL lesions of any kind should, therefore, disrupt these different forms of memory to the same degree.

This distinction between the relative effects of MTL and midline diencephalic lesions on temporal order (and other kinds of contextual) memory and item recognition contrasts with a more recent hypothesis about the functional heterogeneity of amnesia (see Aggleton \& Brown, 1999). This hypothesis, based largely on work with animals, postulates that normal memory is mediated by two systems. The first is the "extended hippocampal system," similar to the circuit of Papez, which com- prises the hippocampus, fornix, mammillary bodies, anterior thalamus, and possibly parts of the cingulate cortex. The role of this system is postulated to be that of linking target information with contextual information such as spatial, temporal, and source information in memory so that particular episodes are uniquely characterised. As such, the "extended hippocampal system" is postulated to mediate memory for all kinds of associations that underlie the process of recollection (item-context retrieval) on which free recall and associative recognition are based. The second hypothesised system comprises the perirhinal cortex and its projection to the dorsomedial nucleus of the thalamus (DM). This system is postulated to mediate the process of familiarity on which recognition of single-item information is based.

According to this dual system hypothesis of global amnesia, midline diencephalic and MTL lesions should have similar effects to each other on both temporal order memory and item recognition provided each kind of lesion disrupts the "extended hippocampal" and perirhinal cortex-DM systems to proportionally equivalent degrees. The predictions of the hypothesis about the relative effects of hippocampal and larger MTL lesions on these two kinds of memory require further discussion. The hypothesis predicts that patients with isolated hippocampal damage will be relatively unimpaired on item recognition memory as this is should be mediated by their intact perirhinal cortex-DM system. However, it is widely believed that temporal order memory relies on the forms of associative memory that underlie recollection. For example, Tzeng, Lee, and Wetzel (1979) proposed that temporal information about items can only be constructed with reference to other items in the list and that at encoding, earlier items provide a context to which later items can be related. Similarly, Michon and Jackson (1984) described temporal information processing as establishing a network of associations between a particular item and the position of other items in relation to it within the context in which these appear. As Aggleton and Brown postulate that recollection depends on the "extended hippocampal sytem," selective hippocampal lesions should impair temporal order memory, but leave 
item recognition intact at least when it depends primarily on familiarity rather than recollection.

In patients with larger MTL lesions that affect both the "extended hippocampal system" and the perirhinal cortex-DM system, memory for both single items and their temporal order would be expected to be impaired according to Aggleton and Brown's hypothesis. Neuroanatomical evidence suggests that the hypothesis should predict that temporal order memory will be more impaired than item recognition in these patients. This evidence indicates that the MTL cortices provide two thirds of the input to the hippocampus (Squire, Knowlton, \& Musen, 1993). So although larger MTL lesions should impair item recognition because they will include perirhinal cortex damage, they will also include hippocampal damage which should disrupt temporal order memory more severely because this will be directly impaired by hippocampal damage and further impaired indirectly because the hippocampus will be receiving degraded item information from the damaged perirhinal cortex, from which it must construct temporal order memory.

The central claim of Aggleton and Brown's hypothesis that hippocampal lesions leave item recognition relatively intact needs to be discussed further because it conflicts with the claim, advanced by Squire and his colleagues, that hippocampal as well as MTL cortex lesions disrupt item recognition (Rempel-Clower, Zola, Squire, \& Amaral, 1996). The latter claim is consistent with the hypothesis that amnesia is functionally unitary, at least to the extent that MTL and midline diencephalic lesions disrupt memory in qualitatively the same way, although Squire and his colleagues, on the basis of the MTL's anatomical inputs, also argue that perirhinal and parahippocampal cortex lesions may well particularly affect visual item and spatial memory respectively (Zola-Morgan \& Squire, 1993). The relevant human evidence about the effects of hippocampal lesions on item recognition is somewhat conflicting. Aggleton and Brown (1999) have reviewed much of this evidence, which includes a meta-analysis (see Aggleton \& Shaw, 1996) of a series of studies that administered two memory test batteries to amnesic patients. The first test battery was the Recognition Memory Test (RMT; Warrington, 1984), which measures item recognition, and the second was the Wechsler Memory Scale-Revised (WMS-R; Wechsler, 1981), which primarily measures free recall. Aggleton and Shaw argued that patients in whom damage was limited to the hippocampus, fornix, or mammillary bodies within the "extended hippocampal system" showed a level of RMT performance that was normal and significantly better than that of the amnesic groups in whom damage was more widespread. This occurred despite the fact that the more selectively lesioned patients showed an impairment on the WMS-R verbal and visual memory quotients that was not significantly less severe than that of the amnesics with more widespread damage. Although the item recognition/free recall dissociation may be partially confounded by the recall scores of the more widely damaged amnesics approaching floor levels, it suggests that it may be inappropriate to use item recognition and recall scores as equivalent measures of the severity of amnesia.

Aggleton and Brown's interpretation of their meta-analysis is also consistent with several recent studies of patients with selective damage to the "extended hippocampal system" and a study of amnesics most of whom had more widespread lesions. First, McMackin, Cockburn, Anslow, and Graffan (1995) found that a group of patients who had suffered selective fornix damage showed normal performance on the RMT despite being impaired on verbal and visual recall tests. Second, and perhaps even more dramatically, Hanley, Davies, Downes, and Mayes (1994) found that a patient with a lesion that included the left fornix and anterior nucleus of the thalamus was completely normal on the RMT, but was badly impaired on tests of verbal recall. Strikingly, this patient performed normally on a verbal recognition test that was as difficult for normal subjects as a verbal free recall test on which she was impaired. Third, Baxendale (1997) found that patients with unilateral left hippocampal sclerosis performed relatively normally and similarly to those with unilateral right hippocampal sclerosis on the RMT for words despite being impaired on verbal recall tests. She also found that patients with right or left 
hippocampal sclerosis performed very similarly on the RMT for faces. Fourth, Vargha-Khadem et al. (1997) found that three patients who had suffered early selective bilateral hippocampal damage performed normally on several item recognition tests that included the RMT, despite showing clear free recall deficits. We have found that another patient, YR, who suffered a similar bilateral hippocampal lesion in adulthood, and who is the focus of the current paper, showed a similar pattern of results in a series of over 40 item recognition tests (see Holdstock, Isaac, Cezayirli, Roberts, \& Mayes, 1999; Mayes, van Eijk, Gooding, Isaac, \& Holdstock, 1999). Fifth, in a factor analysis of the scores of 50 amnesic patients who were given several standardised recognition and free recall tests, Hunkin et al. (2000) have found evidence for separate free recall and recognition factors. This strongly suggests that recall and recognition can be disrupted by differently located lesions.

Patients with apparently selective hippocampal damage have not, however, always been found to perform normally on tests of recognition. Reed and Squire (1997) investigated six patients in whom damage was largely limited to the hippocampus and found them to be impaired on a number of recognition tests including the RMT. These results are consistent with the view that lesions to the hippocampus and MTL cortices do not disrupt memory in qualitatively different ways, and that the severity of the memory impairment increases as the extent of MTL damage increases, although MTL cortex damage probably affects memory more (see also Rempel-Clower et al., 1996). This unitary hypothesis about the effects of MTL lesions in conjunction with the view of Squire and his colleagues, described earlier, that MTL as well as midline diencephalic lesions disrupt item recognition and temporal order memory equally, implies that both selective hippocampal and more extensive MTL lesions should also each disrupt these forms of memory equally.

The reason for the discrepancy in the performance of Reed and Squire's patients and those described elsewhere (see earlier) is unclear. It is possible, however, that Reed and Squire's patients had damage outside the hippocampus, not detected by magnetic resonance imaging (MRI), which contributed to their item recognition impairments. Two of the patients had histories of alcohol abuse, one had a history of mild hypertension and renal failure, and another could not undergo MRI because of a cardiac pacemaker. In this case, isolated hippocampal damage was presumed on the basis of his aetiology of anoxia. However, Markowitsch, Weber-Luxemburger, Ewald, Kessler, and Heiss (1997) have found reduced blood flow in widespread brain regions that appeared normal using structural MRI in patients who had suffered anoxia, indicating that isolated hippocampal damage cannot be assumed in patients with this aetiology.

The views of Squire and his colleagues, Parkin and his colleagues, and Aggleton and Brown make different predictions about the relative effects of hippocampal (and other) lesions on item recognition and temporal order memory. First, the view of Squire and his colleagues predicts that hippocampal, more extensive MTL, and midline diencephalic lesions should each disrupt temporal order memory and item recognition to equivalent extents. This unitary view only predicts that temporal order memory will be more impaired than item recognition when any of these lesions is accompanied by frontal cortex damage that further impairs temporal order memory in a relatively selective way. Second, the view of Parkin and his colleagues that MTL and midline diencephalic lesions disrupt memory in qualitatively distinct ways also predicts that both hippocampal and more extensive MTL lesions should both have equivalent effects on temporal order memory and item recognition. It differs from the unitary view, however, in predicting that midline diencephalic lesions will disrupt temporal order memory more than item recognition even when there is no additional impairment of temporal order memory caused by frontal cortex damage. Third, Aggleton and Brown's view predicts that both hippocampal and more extensive MTL lesions should disrupt temporal order memory to a greater extent than item recognition even when there is insignificant damage to the frontal lobes. Further, hippocampal lesions will not disrupt item recognition at all if this mainly depends on item 
familiarity rather than recollection. This view also predicts that midline diencephalic lesions will have the same effect as hippocampal lesions if they only affect structures in the "extended hippocampal system," and the same effect as more extensive MTL lesions if they additionally damage structures in the perirhinal cortex-DM system.

The current study included three experiments, which compared recognition memory for single items and three kinds of temporal order memory tasks in a patient with selective hippocampal damage, YR. Three different temporal order memory tasks were used, which tapped between-list and two forms of within-list temporal order memory (temporal sequencing and order of words in pairs), because performing these tasks may require retrieval of different kinds of associative information. The aim was to identify the effects of selective hippocampal damage on the three forms of temporal order memory and on item recognition in order to determine which of the hypotheses of Squire and his colleagues, Parkin and his colleagues, or Aggleton and Brown gives the most accurate account of the effects of such damage on these forms of memory. As the first two experiments had found that YR's temporal order memory was impaired relative to her item recognition, the third experiment not only continued the examination of YR's word and temporal order recognition, but also explored whether she could recognise word pairs normally. This was done in order to find out more about the kinds of associative memory that underlie performance on the forms of temporal order memory impaired by hippocampal lesions.

\section{EXPERIMENT 1}

The first experiment investigated the performance of YR and her control subjects on a test of word list discrimination, temporal order memory, and word recognition. The unitary hypothesis of Squire and his colleagues predicts that YR should show equivalently severe item recognition and list discrimination memory deficits because structural MRI has revealed no evidence that $\mathrm{YR}$ has sustained structural damage to her frontal lobes, lesions to which might additionally disrupt list discrimination memory according to the hypothesis. The hypothesis of Parkin and his colleagues makes the same prediction because structural MRI has revealed no evidence that $Y R$ has sustained damage to her midline diencephalic structures, lesions to which should disrupt list discrimination memory disproportionately. In contrast, Aggleton and Brown's hypothesis predicts that YR should have relatively intact word recognition if this depends primarily on familiarity, but should have impaired between-list temporal order memory, provided this depends on recollection, because MRI reveals that she has sustained relatively selective bilateral hippocampal damage.

\section{Methods}

\section{Subjects}

This experiment included patient $\mathrm{YR}$ and 10 control subjects matched to YR for gender, age (mean = 59.0, $S D=11.0$ ), and WAIS-R Verbal IQ (mean = 106.3, $S D=8.1$ ). YR's memory became impaired when she was 49 years old following an incident in 1986 in which she received opiate drugs intravenously for the relief of severe back pain. Some hours following administration of these drugs she was found in a confused state by her sister and was admitted to hospital. She underwent a number of examinations including EEG but no definite cause was found for her memory impairment, although it was believed that she may have suffered an ischaemic infarct. Following this incident her memory impairment seemed mild enough to allow her to return to full-time employment as a clerk in a position that she had held for some years. She was, however, later made redundant because of her memory problem and was unable to find further employment for the same reason. Her memory impairment appears to have remained stable since the initial incident. In judging her ability to cope with daily living, it should be appreciated that this ability may be supported by her relatively normal recognition memory, which helps her to manage in the face of her quite badly impaired ability to recall.

MRI was carried out in September 1997 using a 1.5 tesla SIGNA whole-body magnetic imaging 
system (General Electric, Milwaukee, WI). A 3D T1-weighted radio-frequency spoiled gradient echo (SPGR) image revealed a selective lesion affecting the hippocampus bilaterally along its full anterior-posterior extent (see Figure 1). To investigate the neuroradiological data more fully, volume measurements were carried out on a range of structures from the images of YR and those of eight ageand IQ-matched control subjects (see Holdstock et al., in press). Within the MTL, the volume of both the left and right side of YR's hippocampus was over $2 S D$ s below that of her control subjects. Importantly, measurements of the parahippocampal gyrus, including perirhinal, entorhinal and parahippocampal cortices were above the control mean and there was no evidence of pathology. Although the amygdala appeared small, there was no evidence of pathology on structural images. Analysis of YR's MRI also indicated that her frontal lobe structures were intact (see Figure 1) and that grey to white matter ratios were normal. There was some evidence of parietal lobe atrophy, but this was not atypical for a woman of her age because the volume of both YR's parietal lobes did not differ significantly from that of her control subjects (for an illustration of YR's parietal lobes in comparison with that of three of her age-matched control subjects, see Holdstock et al., in press). Finally, there was no evidence that YR had suffered any damage to midline diencephalic structures.

YR's performance on several standardised neuropsychological tests of verbal and visual recognition and recall is shown in Table 1. Her performance on the Doors and People Test, the Adult Memory and Information Processing Battery (AMIPB), and the RMT revealed that she had a severe recall deficit for both verbal and visual material. However, both her verbal and visual item recognition performance appeared completely unimpaired. In contrast, on the WMS-R YR's visual memory index appeared to be normal whereas her verbal memory index was impaired. One possible reason for this discrepancy is that the visual memory index includes two tests of recognition memory (Figural Memory \& Visual Paired Associates), whereas the verbal memory index provides a pure measure of recall. This interpretation is
Table 1. Performance of $Y R$ on standardised tests of intellectual function (NART IQ, WAIS-R) and memory

\begin{tabular}{ll}
\hline Tests and subtests & YR's performance \\
\hline NART IQ & 115 \\
WAIS-R & \\
Full & 100 \\
Verbal & 108 \\
Performance & 97 \\
Doors \& People & \\
People (verbal recall) & $<1$ st percentile \\
Names (verbal recognition) & 99 th percentile \\
Shapes (visual recall) & 1 st-5th percentile \\
Doors (visual recognition) & 50 th percentile \\
$R M T$ & \\
Words & 45 (50-75th percentile) \\
Faces & 48 (>95th percentile) \\
$W M S-R$ & \\
Verbal & 62 \\
Visual & 102 \\
General & 66 \\
Delay & 72 \\
Attention/concentration & 122 \\
$A M I P B$ & \\
List learning (A1-A5) & below cut-off \\
Story recall (imm) & below cut-off \\
Story recall (delay) & below cut-off \\
Design learning (A1-A5) & $<10$ th percentile \\
Figure recall (imm) & below cut-off \\
Figure recall (delay) & below cut-off \\
\hline & \\
&
\end{tabular}

consistent with YR's performance on the other standardized tests of visual free recall, on which her performance fell below either the 10th or the 5 th percentile.

YR also completed the Cognitive Estimates Test (CET; Shallice \& Evans, 1978), the Verbal Fluency Test (Benton, 1968), and the Wisconsin Card Sorting Test (WCST) in order to examine her executive function. Her performance on these tests gave no evidence that she had an executive function problem. On the WCST, having rapidly identified the three simple sorting rules, she attempted to use other more complex rules for the remaining categorisations and so only achieved three categories (610th percentile). However, she showed a minimal tendency to make perseverative responses (6 responses, 88th percentile). Such perseverative responses are regarded as providing the clearest evidence of executive dysfunction caused by frontal lobe lesions. Her performance on the WCST, 


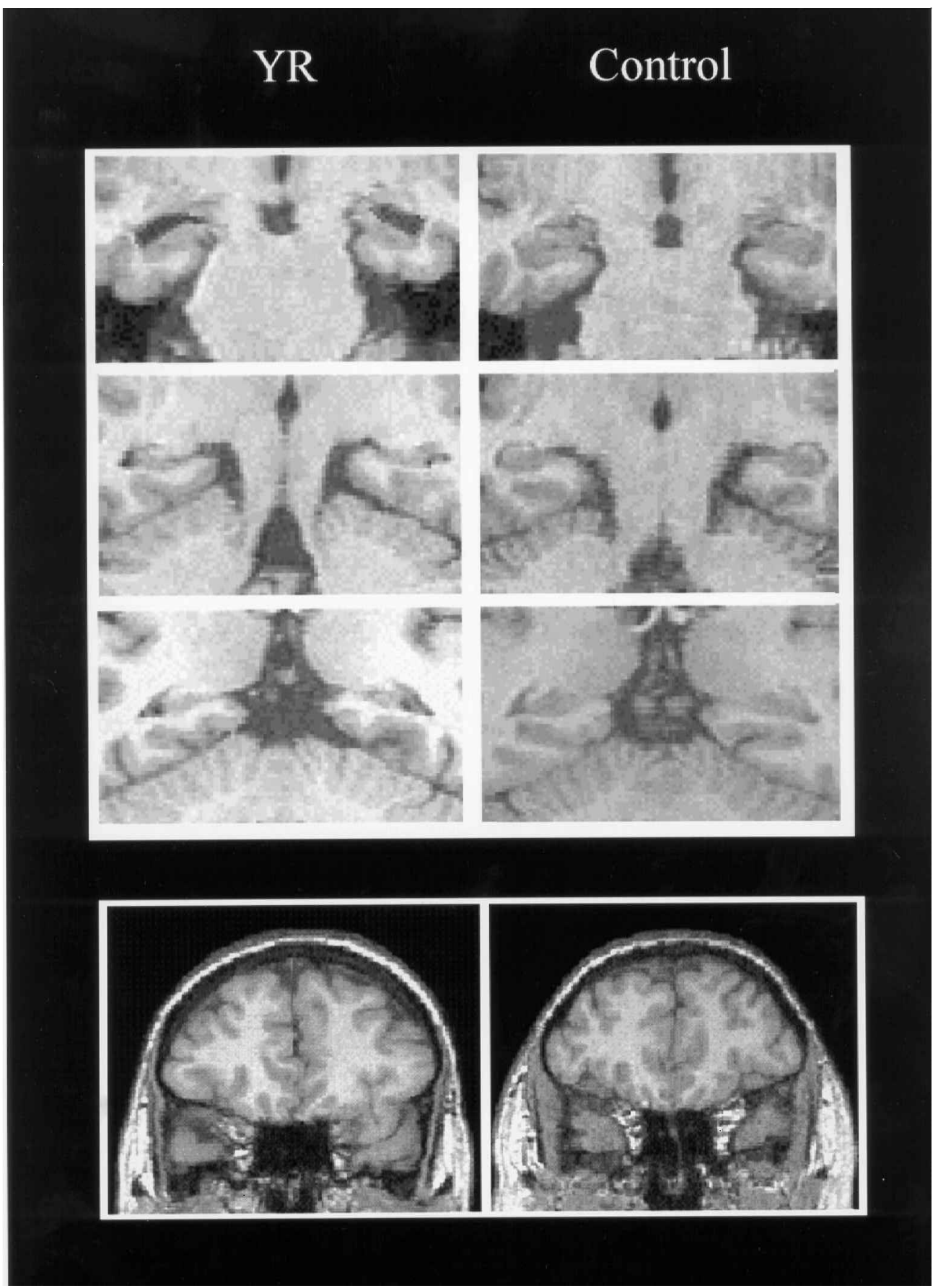

Figure 1. The top three pairs of MRI scans (YR on the left and a matched control subject on the right) show coronal sections through the head, body, and tail of the hippocampus. These scans indicate that YR's hippocampus is damaged along its entire anterior-posterior extent whereas the adjacent MTL cortices are intact. The lower pair of MRI scans show coronal sections through the frontal lobes of YR and the same matched control, illustrating that this region is intact in $Y R$. 
therefore, provided no evidence either that YR perseverated or that she was unable to switch hypotheses, two hallmarks of dysexecutive function caused by frontal lobe damage (see Lezak, 1995). YR's FAS and CET performance was unimpaired compared with that of a group of matched female control subjects (mean age $=59.6, S D=3.6$; mean NART-R IQ = 104.8, $S D=7.2$ ). She scored 0.11 and $.69 S D$ s below the mean level of this control group on the FAS and CET respectively.

\section{Materials}

There were three list discrimination tasks, each comprising two 12-word lists. These were constructed from a total of 288 low-frequency nouns, selected from the Thorndike-Lorge word list. The selected words were divided into 2 sets, $\mathrm{A}$ and $\mathrm{B}$, of 144 words each, matched for word frequency and word length. Each of these lists was divided into 2 subsets of 72 words, designated as targets and distractors, matched on the same criteria. Designation as targets or distractors was fixed for sets $A$ and $B$ across all subjects. Each set of 72 target words was further divided into 3 experimental groups of 24 words, each of which was split into 2 equal length lists of 12 words. Individual subjects were given either set $\mathrm{A}$ or set $\mathrm{B}$, using an alternating sequence. Recognition memory for each set of 24 words was tested using a 2-choice forced-choice procedure, in which individual target-distractor pairs were matched on word frequency and word length.

\section{Procedure}

This experiment was part of a larger one involving global amnesics, a critical requirement of which was that all subjects should be performing well on the test of item recognition. In order to achieve levels of item recognition that were $80 \%$ correct or better, the exposure times to the words in the presentation phase were varied slightly across subjects. The decision about the appropriate exposure duration for each subject was based on their performance on background neuropsychological tests. The mean exposure of the control subjects was $1.4 \mathrm{~s}$, ( $\min =$ $0.5 \mathrm{~s} ; \max =2.5 \mathrm{~s} ; S D=0.77)$. YR received an exposure of $2.0 \mathrm{~s}$. Thus, her exposure was well within the range of the control subjects.
The total list duration in this experiment was fixed at $96 \mathrm{~s}$ with an inter-stimulus interval of $8 \mathrm{~s}$. This constraint was used to ensure that the temporal discrimination was of equal difficulty for all subjects in the sense that temporal ratios between the two lists and the delay to test were constant across all subjects. For all subjects, therefore, following presentation of one word, there was a blank interval before presentation of the next word. To prevent subjects from rehearsing during these intervals they were required to perform a second task. In the time remaining, a sequence of seven-digit numbers was presented for $1 \mathrm{~s}$. Following each presentation subjects were required to report as many of the digits as possible. The lags between the last item of the first list and the first item of the second list, and that between the last item of the second list and the start of the test, were also fixed at $77 \mathrm{~s}$ and $10 \mathrm{~s}$, respectively. During these gaps the experimenter engaged in conversation with the subject.

The list discrimination test was run on a computer with all study and test stimuli presented centrally in upper case (font size 24). The order of presentation of study words and test items was fixed across subjects and groups. It was then explained to subjects that they were to be shown 2 lists of 12 words, following which their memory for the words and in which list they had appeared would be tested. The first 12 words were then shown to the subject, followed by a delay of $77 \mathrm{~s}$. Subjects were alerted to each phase of the experiment by a short beep. Memory was tested in two ways. First, memory for every studied word was examined using a twochoice forced-choice recognition test. The position of targets and distractors was counterbalanced. Subjects were asked which of the two words they remembered seeing, and the response was keyed in by the experimenter. Second, the target word was displayed and subjects were required to indicate whether the word had been presented in the first or second list. The list discrimination question was asked regardless of whether subjects had correctly recognised the target word. This ensured that the delays from study to test were kept approximately constant across subjects and groups. The three list discrimination tests were given in a spaced fashion during the same session. 


\section{Results}

In all experiments, YR's score was considered to be impaired if it was more than 1.96 SDs worse than her control group's mean score, giving a type 1 error probability of .05 on a two-tailed test. The results of YR and her control subjects are shown in Table 2. For the forced-choice word recognition memory test, recognition was scored as the proportion of the total set of 72 test words that was correct. Performance on the list discrimination test was scored as the proportion of recognied words that were assigned to the correct list.

YR's item recognition performance was very similar to that of her control subjects and fell just .5 $S D$ s below their mean score. In contrast, her list discrimination performance was at chance and fell 2.5 SDs below the mean score of her control subjects.

\section{Discussion}

In this experiment, YR performed normally on the forced-choice word recognition test when her exposure to each word was well within the range of exposure times given to her control subjects. The control subjects' performance on this task was well below ceiling levels so there is strong evidence that YR's word recognition was intact. Such normal performance is predicted by Aggleton and Brown (1999) when item recognition depends primarily on judging the relative familiarity of target and foil items, because this kind of familiarity memory can be mediated by the perirhinal cortex-DM system without the need for hippocampal support. In contrast, YR's ability to remember in which list correctly recognised words had been presented was at chance and significantly impaired.

Table 2. Performance of control group (mean and SD) and patient $Y R$ (mean) on recognition and list discrimination

\begin{tabular}{llc}
\hline & Recognition & List discrimination \\
\hline Control group & $85.9(5.5)$ & $60.8(4.2)$ \\
YR & 83.3 & $50.0^{\mathrm{a}}$ \\
\hline
\end{tabular}

${ }^{\mathrm{a}}$ Indicates a significant impairment.
The pattern of YR's memory scores is, therefore, consistent with the view of Aggleton and Brown. This view not only predicts her relatively intact word recognition, but also predicts her impaired list discrimination memory because this is likely to depend on recollection. In contrast, the pattern of YR's memory scores is predicted neither by the view of Squire and his colleagues, nor the view of Parkin and his colleagues.

It should be noted that the list discrimination memory task involved a test of cued recall rather than of forced-choice recognition that would have been comparable to the word recognition task. A comparable forced-choice list discrimination recognition test would be impossible to construct, and even if it were possible to construct, any amnesic subject would be nonspecifically disadvantaged because of the load on memory that the test itself would impose. The fact that cued list discrimination was compared with word forced-choice recognition does not affect the predictions of any of the three views. Thus, Squire and his colleagues have argued that recall and recognition are equivalently impaired in patients with hippocampal lesions (Haist, Shimamura, \& Squire, 1992), and the view of Parkin and his colleagues is partially based on the performance of MTL patients on the list discrimination cued recall task. Similarly, the view of Aggleton and Brown is that hippocampal damage disrupts recollection, and it is almost certain that list discrimination cued recall depends on recollection.

Recall tests differ from recognition tests in an interesting way. The cues provided at test are much less complete representations of the target memory for recall relative to recognition. So much more work needs to be performed in order to complete the memory in a recall test. This may be of importance because the neural network model of O'Reilly, Norman, and McClelland (1998), which, like Aggleton and Brown, postulates that hippocampal lesions disrupt recollection, but not familiarity, also postulates that they impair the ability to complete a memory pattern from a partial cue. According to this model, the hippocampus rapidly forms distinct memory representations even of very similar stimuli and is very efficient at completing 
memory patterns from partial cues. These properties are not shared by the MTL cortices or other neocortical structures. The model can, therefore, explain impairments in free and cued recall following selective hippocampal damage. YR's impaired list discrimination could, therefore, have arisen from a failure of memory pattern completion rather than because she failed to store information that was specific to this form of temporal order memory. Item forced-choice recognition probably depends much less or not at all on the hippocampus' special pattern completion ability, and should be preserved to the extent that recognition depends on familiarity memory, which is mediated by the MTL cortices and other neocortical regions according to the model.

It would, therefore, be of interest to determine whether recognition of temporal order information is also impaired in YR in the face of relatively normal item recognition. Such tests can be devised for within-list temporal order memory so the next two experiments examine forced-choice recognition of two variants of this form of temporal order memory. The predictions of the three views considered above remain the same. A selective deficit in within-list temporal order recognition would, however, be of greater interest because the memory pattern completion demands of the item and temporal order recognition tests are closely equivalent and slight. Such a deficit would be more likely to reflect an impairment in the storage of the kinds of information that specifically underlie within-list temporal order memory rather than a failure of memory pattern completion.

\section{EXPERIMENT 2}

This experiment investigated the performance of YR and her control subjects on two tests of item recognition and two tests of temporal order memory for both verbal material, comprising high-frequency nouns, and visual material, comprising patterns photocopied from wallpaper. Item recognition was investigated using two paradigms: (1) five-choice forced-choice recognition; and (2) fivechoice yes/no recognition in which, although test items were presented one at a time, there were four foils for each target item. Temporal order memory was investigated using (1) a within-list temporal sequencing recall task, where subjects were required to arrange the items at test into the sequence in which they were originally presented, and (2) a forced-choice recognition test, in which subjects had to choose the correct order of the items from five alternative orders. This latter test was designed so that both item and within-list temporal order memory could be examined with similar forcedchoice recognition tests. All previous studies have compared item recognition memory with recall of items' within-list temporal order. Experiment 2's procedure allows one to determine whether YR's within-list temporal order memory was impaired for recall only, or for recognition as well, in the face of relatively normal item recognition. Use of both forced-choice and yes/no item recognition tests allows one to determine whether yes/no recognition is more dependent on the intactness of the hippocampus.

The predictions of the hypotheses of Squire and his colleagues, Parkin and his colleagues, and Aggleton and Brown are basically the same as they were for Experiment 1. More specifically, the first two hypotheses predict that item recognition will be as impaired as temporal order memory regardless of whether temporal order memory is tested by recall or recognition. In contrast, Aggleton and Brown predict that whereas recognition of single items should be normal in YR in so far as it depends on familiarity, memory for temporal order, whether tested by recall or recognition, would be expected to be impaired because it depends on recollection in both cases.

\section{Method}

\section{Subjects}

This experiment included patient YR and 12 female age $($ mean $=58.4, S D=5.7)$ and IQmatched control subjects (mean Full Scale WAIS$\mathrm{R} I Q=107.9, S D=10.3$; mean Verbal WAIS-R $\mathrm{IQ}=106.0, S D=12.9$; mean WAIS-R Performance IQ $=109.7 ; S D=10.3$ ). 


\section{Materials}

Words. There were two word recognition tests, one forced-choice and one yes/no. In both of these, memory was tested for 10 target words, each of which had to be distinguished from four semantically related foil words. One of these tests used a forced-choice procedure and the other used a yes/ no procedure. In order to construct these tests, 100 nouns were selected, 5 drawn from each of 20 categories of the Battig and Montague (1969) category norms. This list was split into 2 lists of 50 words, which comprised 5 words in each of 10 categories. One word from each category was randomly designated as the target word and the others as foils. Semantically related words were used in these conditions in order to increase the difficulty of the test so that subjects would not be performing at ceiling levels. Targets and foils in each list were matched for frequency (range 0-680 per million; Kuçera \& Francis, 1967), rank (Battig \& Montague, 1969), and concreteness. One of these lists was used for the forced-choice recognition test and the other was used for the yes/no recognition test.

For the 5-choice forced-choice temporal order recognition task, subjects had to recognise the order of 5 presented words on 20 trials, whereas for the temporal order recall task they had to recall the order of 8 presented words on 5 trials. To construct the relevant word lists, 140 semantically unrelated nouns were selected (frequency of between 1 and $600)$. One hundred of these were randomly allocated to the temporal order recognition condition and 40 to the temporal order recall condition. For the temporal order recognition condition the 100 words were split into 20 lists of 5 items, which were matched for frequency. For the temporal order recall condition, the 40 words were split into 5 lists of 8 items, also matched for frequency.

Patterns. The format of the visual tests was the same as that of the verbal tests described earlier. For this condition, the stimuli comprised black-and-white photocopies of patterns that had been selected from wallpaper patterns. Each pattern measured 4 by 4 $\mathrm{cm}$ and was pasted onto cardboard and laminated. For the item recognition conditions 20 sets of 5 items were constructed. The patterns within each set of five were very similar. One pattern from each set was randomly selected as the target item and the other four items from the set served as foils. The patterns were split into 2 lists of 10 sets. One of the lists was used in the forced-choice recognition condition and the other in the yes/no recognition condition. The forced-choice test comprised 10 cards measuring 5 by $23 \mathrm{~cm}$, each displaying a target pattern and its 4 foils.

For the temporal order recall and recognition conditions 140 further patterns were selected. One hundred of these were assigned randomly to the temporal order recognition condition and 40 to the temporal order recall condition. As for the verbal task, the patterns in the temporal order recognition condition were split into 20 lists of 5 items and those in the temporal order recall condition were split into 5 lists of 8 items. The temporal order recognition test was constructed in the same way as for the verbal test described earlier. The test was presented on cards measuring 23 by $23 \mathrm{~cm}$. The target sequence of patterns and its four foil sequences were arranged horizontally on each card so that the first item of each sequence was on the left and the last item of each sequence on the right.

\section{Procedure}

All subjects received the same order of presentation. In order to minimise the effects of interference, testing took place in three sessions, with presentation and test of both words and patterns in each. In the first session, subjects completed the item recognition tests, in the second session they completed the temporal order recognition tests, and in the final session the tests of temporal order recall were administered. For all tests, a short filled delay of $15 \mathrm{~s}$ was interposed between the end of the presentation phase and the onset of the test phase in order to minimise the effects of short-term memory on performance.

All tests involving words were presented via computer. Subjects were given a practice run of the distractor task that would be used during the delay between study and test. This was an odd/even judgement task in which subjects read aloud a twoor three-digit random number that appeared on the 
screen and indicated as quickly as possible whether it was odd or even. The rate at which subjects were able to perform this task was stored and the numbers were presented at this predetermined rate during the delays throughout the experiment. The same distractor task was used for both word and pattern conditions. Subjects were then instructed about what the experiment would involve and how their memory would be tested. After the instructions had been given, the words were presented to the subjects for $1 \mathrm{~s}$ each, after which they completed the odd/even judgement task for $15 \mathrm{~s}$. The word forced-choice test, in which each target word was displayed with its four foils in a vertical arrangement on the screen, was then presented. Test items were presented in a random order, which did not correspond with the order of presentation of the targets in the presentation phase. The position of the target item was varied systematically so that it occurred equally often in each position. For each test item, subjects selected which of the words they thought had appeared in the list. If unsure, subjects were encouraged to guess. No time limit was imposed on subjects in the test phase.

Following presentation of the forced-choice recognition test for words, the forced-choice recognition test for patterns was administered. Subjects were instructed about what the experiment involved and how memory would be tested. The pattern forced-choice recognition test was administered after subjects had been given these instructions. Apart from using card rather than computer presentation and a 2 rather than a $1 \mathrm{~s}$ study exposure to the patterns, the procedure for this test was identical to that used for the word forced-choice recognition test.

The yes/no recognition tests were then administered. For these tests, item presentation and distraction conditions for both words and patterns were identical to that of the forced-choice recognition tests. For the yes/no recognition tests, the 10 targets were mixed randomly with the 40 distractors with the constraint that no more than 2 targets appeared next to each other in the list. The order of items at test did not correspond with the order at presentation. The items were presented one at a time to the subjects and, for each item, sub- jects had to decide whether it had occurred in the presentation phase.

In the second testing session, the temporal order forced-choice recognition tests were given. Temporal order recognition was tested for 20 temporal sequences of both words and patterns. In order to minimise the effects of interference, testing occurred in blocks of 10 with presentation and test of 5 of the verbal items followed by presentation and test of 5 visual items. There was a break of a few minutes between blocks. Before the tests were given, subjects were instructed about what each experiment involved and how memory would be tested. The procedure for the temporal order recognition tests of the words and patterns was identical except that the patterns were presented on cards rather than on the computer and were each presented for $5 \mathrm{~s}$ at study, unlike the words, which were each presented for $4 \mathrm{~s}$. Thus, for both tests, 5 items were presented one at a time. Subjects were required to remember both the items in the list and the order in which they were presented. After completing the $15 \mathrm{~s}$ distractor task, 5 lists of items were presented together. Each of these lists contained the same 5 items subjects had just studied, but these items were presented in the correct order in only one of the lists. Subjects had to indicate which list was in the correct order.

The foil lists were constructed systematically to minimize the use of strategies based on remembering the first or last item. For half of the 20 test items, the first item remained consistent across targets and foils. In addition, either one or two of the foils shared the final item with the target. The three middle items were varied pseudorandomly to be similar to the target. For the other half of the 20 items, the last item was held constant across targets and foils and either one or two foils shared the same initial item.

In the third testing session, the temporal order recall test was given. Presentation was identical to that used for the temporal order recognition test, but following performance of the distractor task in both verbal and visual conditions, a series of cards with one item on each card was arranged in front of the subject. For each set, the items were arranged in a different order to the presentation order so that 
there was a near zero correlation between presentation order and the order in which the cards were placed before the subject during the test phase. For both verbal and visual tests, subjects had to rearrange the cards to be as close to their original order as possible.

\section{Results}

The results of the verbal memory tests for YR and her control subjects are shown in Table 3. On the word forced-choice recognition test, YR's performance was well within the range of scores of the control subjects and 1.1 SDs below their mean. The results of the word yes/no recognition test were analysed using signal detection theory (SDT). It can be seen from the table that YR's d' score fell exactly $2 S D$ s below that of the control mean, indicating that her performance was impaired when assessed by yes/no recognition for this material.

YR's performance on the forced-choice recognition test for temporal order was then assessed and was found to be over 3.3 SDs below that of the control subjects. Temporal order recall was scored in two ways. First, Spearman's rho correlations were carried out between the original order of the items and the order produced by the subjects. This correlation was averaged across the five items for each subject. Second, a measure of serial organisation was calculated (see Mangels, 1997). For this measure, subjects were given one point for every pair of items that occurred in the correct order. As there were eight items in the test the maximum score was, therefore, seven. From the table it can be seen that YR's recall of temporal order was impaired using both of these methods of analysis (on the correlational measure her performance was 4.0 SDs below that of her controls and on the serial organi- sation measure it was 3.5 SDs below that of the controls).

The results obtained with the visual tests were then analysed in the same way as the verbal tests. The results obtained by YR and her control subjects are shown in Table 4. On the forced-choice test of item recognition YR's performance was 3 SDs above her control group's mean score. On the yes/ no test, in contrast to her performance with the verbal material, her performance fell only .4 SDs below the controls' mean and well within their range.

On the forced-choice temporal order recognition test, YR's score fell over 2.0 SDs below the mean of the control subjects and was, in fact, outside the range of this group indicating a significant impairment on this task. Temporal order recall was examined using the two measures described earlier for the verbal tests. First, the correlational measure indicated that YR's temporal order recall was 5.0 $S D$ s below that of her control subjects. Using the serial order measure the performance of all subjects was lower than that with the verbal material and it was not possible to demonstrate a significant impairment in YR's performance because the performance of the control subjects was not 2 SDs above floor. Therefore, although YR's score on this measure was at floor and 1.7 SDs below that of the control subjects, it was not possible to demonstrate a significant impairment.

\section{Discussion}

YR's performance on the visual memory tests is relatively easy to interpret. She showed normal recognition memory for the patterns whether tested by forced-choice or yes/no tests. This is consistent with her performance on a large number of other

Table 3. Performance of control group (mean and SD) and patient YR (mean) on verbal tests

\begin{tabular}{|c|c|c|c|c|c|}
\hline & \multirow{2}{*}{$\begin{array}{l}\text { Item Forced } \\
\text { Choice }\end{array}$} & \multirow[b]{2}{*}{ Yes/no d' } & \multirow{2}{*}{$\begin{array}{c}\text { Temporal } \\
\text { order recognition } \\
\text { Raw score }\end{array}$} & \multicolumn{2}{|c|}{ Temporal order recall } \\
\hline & & & & Correlation & Serial order \\
\hline Control group & $9.2(1.1)$ & $2.4(0.8)$ & $16.0(2.7)$ & $0.9(0.1)$ & $4.5(1.0)$ \\
\hline YR & 8.0 & $0.8^{\mathrm{a}}$ & $7.0^{\mathrm{a}}$ & $0.5^{\mathrm{a}}$ & $1.0^{\mathrm{a}}$ \\
\hline
\end{tabular}

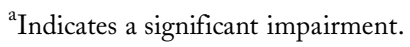


Table 4. Performance of control group (mean and SD) and patient YR (mean) on visual tests

\begin{tabular}{|c|c|c|c|c|c|}
\hline & \multirow{2}{*}{$\begin{array}{c}\text { Item Forced } \\
\text { Choice }\end{array}$} & \multirow[b]{2}{*}{$Y e s / n o d^{\prime}$} & \multirow{2}{*}{$\begin{array}{l}\text { Temporal } \\
\text { order recognition } \\
\text { Raw score }\end{array}$} & \multicolumn{2}{|c|}{ Temporal order recall } \\
\hline & & & & Correlation & Serial order \\
\hline Control group & $8.6(1.4)$ & $1.6(0.5)$ & $16.1(2.5)$ & $0.8(0.1)$ & $2.8(1.5)$ \\
\hline YR & 9.0 & 1.4 & $11.0^{*}$ & $0.3^{\mathrm{a}}$ & 0.2 \\
\hline
\end{tabular}

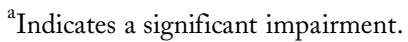

visual item recognition tests at which she has typically performed normally (Mayes et al., 1999). Strikingly, YR's score on the forced-choice recognition test was higher than her control group's mean score, and higher than or equal to the score of 8 of her 12 control subjects.

On the tests of temporal order memory for the patterns, YR was clearly impaired not only on the recall, but also on the recognition test. Her performance on the serial order measure of recall was at chance, although this measure could not be used to demonstrate an impairment given the control subjects' poor performance. She was, however, greatly impaired on the correlational measure of recall so there is little reason to doubt that her ability to recall the presentation order of the patterns was severely compromised. This finding is not surprising, however, because YR is impaired at all forms of recall whether for items or for more complex information. Her performance on the recognition test for the order of the patterns is most appropriately compared with her performance on the forced-choice recognition test of the patterns because both tests were of the forced-choice kind. It is possible that these tap slightly different memory processes from yes/no recognition tests at least under some conditions, although no support is given to this possibility from YR's performance on the pattern recognition tests. The comparison makes clear that YR was impaired at recognition of the temporal order in which patterns were presented although she could recognise the patterns themselves normally. Aggleton and Brown's hypothesis predicts this on the assumption that item recognition depends mainly on familiarity whereas temporal order recognition as well as recall involves recollection. Although the deficit is not severe (YR's performance is well above chance), it is likely that the severity of the deficit would be greater when mem- ory load is increased, i.e., recognition of the order of longer lists is tested.

Interpretation of YR's performance on the verbal memory tests is somewhat trickier to interpret because there is evidence that her yes/no recognition of words was significantly impaired. This was not true, however, of her forced-choice word recognition, which fell within the normal range. In contrast, her recognition of the temporal order of presentation of the words fell over $3.3 \mathrm{SD}$ s below that of her control group. It is reasonable to argue, therefore, that YR was more impaired at recognising within-list temporal order of words than she was at word recognition (which was not impaired) when both tests were of the same kind (i.e., forced choice).

Two counterarguments to this proposal need to be considered. First, it might be countered that a significant deficit on forced-choice word recognition was being concealed by a ceiling effect in the control subjects. This is possible, but perhaps unlikely, because YR typically is not impaired at verbal item recognition tests where ceiling effects cannot be involved (see Table 1, Table 2, and Mayes et al., 1999). Furthermore, YR's word forced-choice recognition score was equal to or better than that of 4 of her 12 control subjects.

Second, the proposal does not explain why word yes/no recognition was impaired in YR. The answer must remain speculative at the moment, but one should not assume that selective hippocampal damage will leave performance on all tests of item recognition intact. It is possible that performance on the yes/no word recognition test depends on a form of recollection as well as on familiarity at least when targets and foils are very similar. In contrast, performance on the forced-choice word recognition test or the pattern recognition tests may depend solely on item familiarity even when targets 
and foils are very similar (see O'Reilly et al., 1998). If Aggleton and Brown (1999) are correct, then hippocampal damage should disrupt recollection, but leave familiarity intact.

If this interpretation is correct, then the word memory results are also consistent with Aggleton and Brown's hypothesis. According to this hypothesis, if deficits in the recognition and recall of within-list temporal order information occur, as they do in YR, then they must be caused by the disruptive effects of hippocampal damage on the forms of associative memory that are critical for recollection. Moreover, whereas the within-list temporal order recall deficits could arise because of a difficulty in completing memory patterns from partial cues following hippocampal damage (see O'Reilly et al., 1998), this possibility is a far less likely explanation of YR's temporal order recognition deficit. In other words, the recognition deficit for within-list temporal order, but not item information, most likely arose because hippocampal damage prevented the storage of information relevant for this form of temporal order memory, but did not prevent the storage of information that was sufficient for normal performance on item forcedchoice recognition tests. This strongly suggests that temporal order memory depends on storing and retrieving kinds of association distinct from and additional to the intra-item associations that presumably are critical for item recognition. The next experiment will examine further the kinds of association that have to be stored and retrieved for successful within-list temporal order memory to occur.

\section{EXPERIMENT 3}

The evidence of the first two experiments indicates that selective hippocampal damage disrupts both a form of between-list temporal order memory and a form of within-list temporal order memory. But it remains unclear what kinds of associative memory need to be stored and retrieved in order to succeed at these forms of temporal order memory. Aggleton and Brown's hypothesis provides little guidance because it proposes that selective hippocampal damage impairs all forms of associative memory that relate to recollection.

There is evidence, however, that selective hippocampal damage does not impair all kinds of associative memory. Vargha-Khadem et al. (1997) have reported that their three patients with selective bilateral hippocampal damage performed normally not only on several tests of item recognition, but also on forced-choice recognition tests of word pairs and face pairs where successful performance depended crucially on identifying which pairs went together at study. The patients were impaired, however, on forced-choice tests of object-place and voice-face associations which tap memory for associations between components of different kinds. We have shown that YR is also impaired at recognition memory for object-location and voice-face associations, as well as at recognition of animal picture-occupation name associations (see Mayes et al., 1999). If item familiarity memory depends on forming or strengthening associations between the components of the items, then these results suggest that selective hippocampal damage spares the consolidation into memory of intra-item associations (e.g., the components of faces) or between items or components of similar kinds (faces or words). Such associations are within-region in the sense that their components are probably represented within one neocortical region. In contrast, the results suggest that selective hippocampal damage disrupts consolidation into memory of associations between different kinds of component (e.g., objects and their location, or faces and voices). Such associations are cross-region in the sense that their components are probably represented in distinct neocortical regions. In other words, the evidence suggests that hippocampal damage disrupts crossregion, but not within-region, associative memory. If this is correct, then the Aggleton and Brown hypothesis will need to be modified.

The third experiment had several aims. First, it sought to determine whether YR was unimpaired at forced-choice recognition of word pairs (like the patients of Vargha-Khadem and her colleagues) whilst she was impaired at a very similar task that assessed forced-choice recognition of the temporal order of the two words in each of a series of studied 
word pairs. The recognition tasks were made very similar so that the major difference between them would be the need to recognise the temporal order of presentation of studied items. Finding that temporal order, but not word pair recognition, was impaired in YR would indicate that although within-list temporal order recognition may depend on retrieving within-region associations, their retrieval is not sufficient to support normal temporal order recognition. Such normal recognition must also depend on the retrieval of different kinds of association, memory for which is impaired by hippocampal lesions.

Second, the experiment sought to confirm that YR's forced-choice word recognition was intact even when control subjects perform well below ceiling levels. Support for this deficit pattern would confirm further the selectivity of the temporal memory problems caused by hippocampal damage.

Third, it sought to determine whether the extent of any temporal order recognition deficit shown by YR was a function of whether temporal order information was or was not intentionally encoded. If temporal order recognition improved in normal subjects when such information was deliberately encoded, but YR's recognition was equivalently impaired regardless of whether or not her encoding of temporal order information was intentional, then her temporal order memory deficit would be unlikely to result from an impairment of organised encoding. In turn, this would make it very unlikely that her temporal order memory deficit reflected an executive function problem that was caused by a frontal lobe dysfunction.

There were four tasks: the single word recognition test was carried out before the word pair recognition task, and then the temporal order word pair recognition task with both incidental and intentional encoding conditions was run last. As a slightly different matched control group was used for the word and word pair forced-choice recognition tests than was used for the temporal order recognition tests, testing word and word pair recognition is referred to, for convenience, as the first part of the experiment, whereas testing temporal order recognition is referred to as the second part of the experiment.

\section{Method}

\section{Subjects}

In the first part of the experiment, YR and 10 control subjects, matched to her on age (mean $=60.4$, $S D=3.5$ ), gender, and verbal intelligence (mean verbal WAIS-R IQ=104.1, $S D=7.6$ ), were compared. In the second part of the experiment, YR was compared to a different group of nine control subjects, matched to her on age (mean $=59.9, S D=$ 5.1 ), gender, and verbal intelligence (mean verbal WAIS-R IQ = 106.8, $S D=7.3$ ).

\section{Materials and design}

The four tasks in this experiment were all presented on an Apple Macintosh VDU. For the complete experiment a total of 240 bisyllabic words with frequencies in the range 50-1000 per million were selected (Kuçera \& Francis, 1967). These were divided into 5 frequency-, concreteness-, and word length-matched subsets, 2 comprising 60 words and 3 of 40 words each. The first 2 subsets were used in the construction of the single word recognition test, and the remaining three were used in the construction of the word pair recognition test and the two versions of the word pair temporal order recognition tests.

For the single word recognition test, 1 subset was designated as the 60 target words and the second subset was used as foils in the 2-alternative forced-choice recognition test. For the remaining 3 tests, which all involved word pairs, the 40 word subsets were used to form 20 unrelated word pairs which were used as target items, and which were recombined in different ways to form the test items for the 2 types of recognition test.

In the first part of the experiment, single word recognition and word pair recognition was tested. Single words were tested using forced-choice recognition, which involved presenting the matched target and foil words side by side with an equal probability that targets occurred on the left and right. In the case of word pairs, recognition was tested in the following manner. Each test item comprised three words. One word was shown to the left and two were shown to the right, one above the other. Each left-hand word corresponded to one of 
the left-hand words of the studied pairs, and the two right-hand words corresponded to the righthand words of two studied pairs, one of which had been paired at study with the left-hand word of the test item. The task for subjects was therefore to indicate the original pairing by selecting the appropriate right-hand word. Each left-hand word from the studied pairs was shown once at test, whereas the right-hand words were shown twice, both as a target and a foil selection.

In the second part of the experiment, word pair order recognition was tested. Unlike the word pair recognition test described earlier, word pairs in this case were presented sequentially, one word at a time, temporally bounded by rows of asterisks. In this way, memory for the sequential order of words comprising the pairs could be tested. At test, this was achieved by presenting the words from each pair simultaneously in two alternative forms, one above the other, either with the first word on the left-hand side or the second word on the left-hand side. The left-right ordering, which corresponded to the correct temporal sequencing of the words within pairs, was shown equally often as a top or bottom selection.

\section{Procedure}

The word recognition task was completed some weeks before the word pair recognition task. For the single word recognition task, target stimuli were presented on the computer monitor at a rate of $2.5 \mathrm{~s}$ per word. Subjects were asked to read and to try and remember each word as it was presented. Immediately following the presentation phase, subjects were shown pairs of words (one studied, one unstudied) on the computer screen and asked to indicate which word of each pair they had just been shown. Order of presentation and testing for the words was different, but fixed across subjects.

For the word pair recognition test, the 20 target pairs were also presented at a rate of $2.5 \mathrm{~s}$ per item. Both words of each pair were presented simultaneously in a horizontal left-right format, and subjects were instructed to read the pairs aloud and remember the pairing. In the associative recognition test, which immediately followed the study phase, the left words in each word pair were pre- sented one at a time. To the right of each such word appeared the word with which it had appeared at study and another word which had been differently paired at study. Subjects were asked to indicate which of the right-hand words had been paired with the left-hand word at study. Order of the pairs at initial presentation and testing was different, but fixed across subjects.

The word pair temporal order recognition test was administered a few weeks after the single word and word pair recognition tasks had been completed. Subjects were first given the task with incidental instructions and later the second list with the intentional instructions. The 40 words comprising the pairs were presented 1 at a time in a box in the centre of the screen. Each word remained on the screen for $2.5 \mathrm{~s}$ and subjects were instructed to read each of these words aloud. After every two words a row of asterisks appeared in the box and remained for $2.5 \mathrm{~s}$. The purpose of this was to provide temporal boundaries for the words in each of the pairs. This procedure was repeated so that subjects saw the entire list of words twice, because pilot work had indicated that two exposures would be necessary to avoid floor effects in normal subjects.

In the incidental condition subjects were instructed merely to try and remember all the words they saw. In a forced-choice recognition test, each word pair was shown twice, one above the other, in complementary left-right pairings. In one pairing, the first presented word was shown on the left, and in the other it was shown on the right. Subjects were instructed that temporal order corresponded to the left-right sequence, and were asked to indicate which pairing represented the correct order of presentation.

The study and test procedure was identical for the intentional condition except that subjects were told at study that the pairs of words would be separated by asterisks and that they should try and remember the order of the two words in each of the presented pairs.

\section{Results}

The scores of YR and her control groups from both parts of the experiment are shown in Table 5. In the 
Table 5. Performance of control group (mean and SD) and patient YR (mean) on single word, word pair, and word pair temporal order (incidental, intentional and overall) recognition

\begin{tabular}{llllll}
\hline & $\begin{array}{c}\text { Forced-choice } \\
\text { single word } \\
\text { recognition }\end{array}$ & $\begin{array}{c}\text { Forced-choice } \\
\text { word pair } \\
\text { recognition }\end{array}$ & Incidental & Intentional & Overall \\
\hline Control group & $47.1(3.6)$ & $15.0(3.0)$ & $14.0(1.7)$ & $\begin{array}{l}\text { Forced-choice word pair } \\
\text { temporal order recognition }\end{array}$ \\
YR & 46 & 14 & 11 & $12^{\mathrm{a}}$ & $11.9)$ \\
\hline
\end{tabular}

${ }^{a}$ Indicates a significant impairment.

first part of the experiment, it is clear that YR's single word and word pair recognition scores were close to the mean of her control group. In neither the single word nor the word pair recognition tasks were the control subjects scoring anywhere near ceiling levels. Also, with both the single word recognition and word pair recognition tasks, more than half the control group scored at or below the level achieved by YR.

In the second part of the experiment, YR's overall temporal order recognition score, based on both incidental and intentional encoding conditions, was $2.3 S D$ s below the mean level of her control group. Her recognition in the intentional encoding condition was $2.1 S D$ s below the mean level of her control subjects whereas her recognition in the incidental encoding condition was $1.7 \mathrm{SDs}$ below the mean level of her control subjects. YR was clearly close to floor in both encoding conditions, particularly after incidental encoding, so her deficits are probably underestimates, and do not differ from each other. The control subjects showed significantly better temporal order recognition after intentional encoding $(t(8)=-2.91, p=.02)$, and YR scored slightly better in this encoding condition although her results are difficult to interpret because they are clouded by her floor-level performance.

\section{Discussion}

The results of this experiment support the view that YR is not usually impaired at forced-choice recognition memory for single words. In none of the three forced-choice word recognition tests of YR and her control subjects that have been reported in this paper did YR show a significant impairment. Her impairment on the five-choice yes/no recognition task of Experiment 1 remains unexplained, but one possibility is that successful performance on this task required the recollection of associations between the words and aspects of their study context. Aggleton and Brown's hypothesis postulates that hippocampal lesions should impair this kind of memory. In contrast, all the other recognition tasks of single items reported in this paper may have been mediated solely by identifying the relative familiarity of the studied materials, and Aggleton and Brown postulate that this form of memory is unimpaired by hippocampal lesions. The word yes/no recognition test not only had many foils, but these were similar to the target words and may have shown very little difference in relative familiarity because even the unstudied foils would have had appreciable familiarity. In contrast, the yes/no pattern recognition task at which YR performed normally included foils that may have had very different levels of familiarity to those of the studied targets because all the patterns were novel prior to the study episode. Hence, the patterns would probably have acquired much more familiarity from study than the words did in the yes/no word recognition task.

Like Vargha-Khadem et al.'s (1997) three hippocampal patients, YR also showed normal associative recognition of word pairs. We have shown that this is also true for her forced-choice recognition of face pairs, as Vargha-Khadem et al. reported for their three patients (see Holdstock et al., 1999). Why forced-choice recognition of these kinds should not be disrupted by selective hippocampal damage is considered further in the General Discussion. 
In contrast to her recognition of the word pairs, YR was impaired at recognising the temporal order of the two words in each of a sequentially presented series of word pairs. Indeed, her performance differed little, if at all, from chance levels. The extent of her problem was not less when subjects did not intentionally encode this form of temporal order, but merely concentrated on remembering the words. YR was, therefore, not only impaired at recognising the temporal order of five sequentially presented items, but was also impaired at recognising the order of presentation of items within pairs. This last deficit is particularly striking because YR performed normally on the forced-choice word pair recognition task. Her pattern of memory deficit indicates that she can recognise which two words went together at study, but not in what temporal order they were shown. This dissociation is unlikely to be determined by temporal order recognition placing greater demands on memory pattern completion (see O'Reilly et al., 1998), because performance on the word pair and temporal order memory tasks is likely to require similar and small amounts of pattern completion. The significance of this dissociation will be considered further in the General Discussion, as will be the significance of the finding that her temporal memory deficit was not reduced when subjects were not specifically instructed to encode the temporal order of the two words in a series of word pairs.

\section{GENERAL DISCUSSION}

The results of the first two experiments support the view that selective hippocampal damage causes deficits in memory for within-list and between-list temporal order memory, but usually spares recognition of single items. The third experiment provides further support for this and suggests that hippocampal damage disrupts recognition for the order of presentation of the two items in a series of presented item pairs even though it does not impair recognition of item pairs as well as single items. Before considering the implications of this interpretation further, two alternative views, which attribute YR's pattern of memory deficits to poten- tial damage in other brain regions, as well as the possibility that the pattern arises simply because the impaired tasks are more difficult, will be considered.

The first alternative view is that YR's impairments in temporal order memory were caused by frontal neocortex damage rather than hippocampal damage. As discussed in the Introduction, deficits in memory for temporal order in the face of intact item recognition have been reported to be caused by frontal lobe damage (e.g., Shimamura et al., 1990). Such damage disrupts executive processing so that information is likely to be suboptimally organised at encoding or retrieval when memorising is intentional. This is likely to have little effect on item recognition, which is minimally affected by elaborative processing, but is very likely to disrupt the encoding and retrieval of the more complex associations likely to be critical for temporal order memory provided that memorising is intentional. Evidence that temporal order memory in patients with frontal lobe damage improved to normal levels when dependence on strategic encoding and attentional effort was minimised (e.g., Milner et al., 1991) supports the view that the functional deficit is one of planned processing and not of storage of associations relevant to temporal order memory. This view is also supported by a study which found that patients with frontal lobe damage were impaired at within-list temporal order memory for semantically related words when encoding was intentional, but not when it was incidental (Mangels, 1997). Unlike their control subjects, the patients were unable to stop encoding the words in semantic clusters when instructed to remember the words' order of presentation.

Although frontal lobe damage can impair temporal order memory, it is most unlikely that such damage explains YR's pattern of performance on the memory tests described in this paper. Careful analysis of YR's structural MRI scans provide no evidence that she has suffered any damage to her frontal lobes. This finding is consistent with her normal performance on several tests of executive function (see Table 1). Also, consistent with the normal appearance of YR's frontal lobes was the finding in the third experiment that her deficit in 
recognising the order in which the two words in a series of sequentially presented word pairs appeared was as great when there was no instruction to remember temporal order as it was when there was such an instruction. YR's control subjects' temporal order recognition improved significantly following intentional encoding, which supports the view that directed encoding of temporal order information is beneficial. Although YR showed slightly less improvement than did her control subjects, the difference was not significant and may simply have been a reflection of the fact that she was performing close to floor levels in both incidental and intentional conditions. As directed encoding is likely to be mediated by the frontal lobes, it seems likely that patients with frontal lobe damage should only be impaired when they are given intentional instructions because they are unable to use executive processes efficiently to enhance the encoding of the temporal order of the words in each pair. This was the case with a very similar temporal order memory task for the frontal lobe damaged patients of Mangels, as described earlier.

The second alternative view is that YR's pattern of memory impairment is caused by parietal lobe damage. Although to our knowledge there is currently no published evidence that parietal lobe damage of any kind ever causes a syndrome like global amnesia or the type of memory deficits that may be produced by frontal lobe damage, the parietal lobes have often shown blood flow increases during neuroimaging studies of memory (see Cabeza \& Nyberg, 1997). Indeed, one study has reported greater blood flow increases in this region during order recognition than during item recognition (Cabeza et al., 1997). It remains to be shown, however, that patients with lesions closely coterminous with the activated parietal regions are impaired at temporal order recognition to a greater extent than they are at item recognition. This is important because the activations may be incidental to successful temporal order recognition, reflecting either unrelated processes or at least processes that are not critical for normal performance. Even if the key role of parts of the parietal cortex in temporal order, but not item, recognition is confirmed, such damage is very unlikely to be the explanation of
YR's temporal memory deficits. As already indicated, YR's parietal cortex atrophy is within the range of what is typical for a woman of her age. Furthermore, very mild nonfocal atrophy is much less likely to disrupt temporal order memory significantly than focal damage, which selectively destroys the putatively critical parietal cortex region.

YR's pattern of impaired temporal order memory and contrastingly relatively normal item recognition memory was, therefore, very probably caused by hippocampal rather than parietal or frontal cortex damage. Some might argue, however, that she was more impaired at the temporal memory tasks simply because they were more difficult than the item recognition tasks. If difficulty is operationally defined as level of performance on a task shown by normal subjects, however, this explanation is very unlikely to account for the results with pattern temporal order recognition in Experiment 2 and word pair temporal order recognition in Experiment 3. In these comparisons, item recognition, word pair recognition, and temporal order memory scores were quite closely matched in the control subjects. The control subjects found temporal order recognition slightly harder than item recognition with words in Experiment 2, and list discrimination much harder than word recognition in Experiment 1, so difficulty remains a possible explanatory factor in these comparisons. Possibility is not actuality, however, and it is worth noting that YR has performed normally at item recognition tasks even when normal subjects find such tasks harder than associative recognition tasks at which she is impaired (see Mayes et al., 1999). So there is no clear evidence that difficulty per se is sufficient to produce impaired memory performance in YR. More probably, her failure relates to deficits in specific memory processes, the nature of which require some further discussion.

YR's results are incompatible with the view of Squire and his colleagues that amnesia, caused by either MTL or midline diencephalic lesions, is a disorder that always includes significant item recognition deficits. According to this view, all patients with these lesions will always show recognition as well as recall deficits, and these deficits should probably be of equal severity. In contrast to this predicted pattern, YR rarely shows impair- 
ments on item recognition tests although she is as impaired at free recall as a patient with extensive MTL lesions to whom she has been compared. This patient was severely impaired at item recognition memory (see Holdstock et al., in press). Aggleton and Brown (1999) have proposed that the relatively normal item recognition found after hippocampal lesions arises whenever recognition can be mediated successfully by familiarity memory alone. They propose that this form of memory is preserved following selective damage to the "extended hippocampal system" and can be mediated by the intact perirhinal cortex-DM thalamus system.

It is less clear, however, whether intact familiarity is sufficient to explain the preservation of wordword associative recognition memory that we have found in YR and Vargha-Khadem et al. (1997) found in their hippocampally lesioned subjects. It has been argued that this kind of recognition mainly relies on recollection (Yonelinas, 1997). It could be, however, that word pair recognition only depends significantly upon recollection under a limited set of conditions. These conditions will need to be specified. If this can be done, it remains possible that, when these conditions are not present, recollection does not enhance recognition performance above the levels that can be achieved by judging the relative familiarity of studied and unstudied word pairs. Although previously not seriously considered, this possibility warrants careful assessment given the findings with patients who have selective hippocampal lesions. If it is found to hold, then hippocampal damage would leave word pair familiarity intact, but impair the ability to associate in memory such word pairs with aspects of the context in which they were studied.

In contrast to her item recognition memory, YR's recognition and cued recall memory for various forms of temporal order information were impaired. The same is true for her recognition of allocentric spatial information (Holdstock et al., 2000). This is inconsistent with one aspect of the view of Parkin and his colleagues. According to this view, MTL lesions should disrupt temporal (and spatial) memory to the same extent as item recognition memory. This is clearly not true of $\mathrm{YR}$, who is more impaired at temporal order memory than she is at item recognition, at which she was relatively intact. In unpublished work, we have also found evidence that patients with MTL lesions that extend into the MTL cortices were also more impaired at between-list temporal order memory than they were at item recognition. This is also inconsistent with the view of Parkin and his colleagues that MTL lesions should disrupt item recognition and temporal order memory to the same extent. It remains to be determined, however, whether the degree to which the temporal memory deficit is disproportionate to the item recognition deficit is equivalent in patients with extensive MTL and midline diencephalic lesions who have equally severe item recognition deficits. If midline diencephalic patients do have relatively more disproportionately severe temporal order memory deficits than MTL patients, then Parkin and his associates could still be correct in proposing that lesions in the two sites disrupt memory in a different way.

Given our and Vargha-Khadem et al.'s (1997) finding that hippocampal lesions seem not to disrupt recognition of associations between information of the same kind (e.g., word pairs), it is likely that memory for such within-region associations are necessary, but cannot be sufficient for withinlist temporal order recognition. Rather, the forms of within-list temporal order memory for which YR shows impaired forced-choice recognition must also depend critically on recognising cross-regional associations at which YR and Vargha-Khadem et al.'s patients are impaired. Such a deficit in recognising associations like those between objects and locations, and between faces and voices, suggests that selective hippocampal damage impairs recognition of associations between different kinds of information, the components of which are likely to be represented cross-regionally. These associations can either be between different kinds of item (e.g., faces and voices), link a single item to a relationship (an object to its location), or link similar items with a relationship (the relative position of two words). The recognition of the temporal order of the two words in each of a series of presented word pairs is an association of this last kind. This form of 
memory involves linking two words by a specific kind of relationship rather than merely linking them arbitrarily. Furthermore, if the reasoning is correct, the temporal relationship cannot be a simple verbal property represented in the same neocortical region as the words, but must be represented in a different neocortical region. It remains unspecified, however, whether there is a specific kind of relationship that uniquely identifies temporal order or whether this relationship necessarily also helps define nontemporal properties. Whether or not the relationship is specific to temporal order, it seems very likely that recognition of the temporal order of a series of items within a list also depends on forming and retrieving the kind of crossregional associations described earlier.

It is unclear whether this argument can be extended to cued recall of list discrimination information because YR's impairment at this kind of memory may solely have resulted from a failure of memory pattern completion (see O'Reilly et al., 1998) and need not indicate that the information required to be retrieved was any different from that involved in representing items (i.e., within-region associative information). However, the betweenlist and within-list forms of temporal memory are relatively similar so retrieval of similar kinds of cross-regional associations may be involved with both. This matter may be resolved through the use of appropriate functional neuroimaging procedures.

It is plausible to propose that the hippocampus is vital for recognition of associations between different kinds of information likely to be represented in distinct neocortical regions, whereas it is not vital for recognition of either intra-item associations or associations between similar kinds of information likely to be represented in one neocortical region. For example, Mishkin, Vargha-Khadem, and Gadian (1998) have argued that there is highly interconnected hierarchy of MTL structures, with the hippocampus lying at the apex of the hierarchy and the parahippocampal, perirhinal and entorhinal cortices lying slightly lower down. Information from sensory cortices is projected to the perirhinal, and parahippocampal cortices in the MTLs. These cortices provide the major input to the entorhinal cortex which, in turn, provides the major source of input to the hippocampus. As sensory information progresses up this hierarchy, it is hypothesised to undergo greater convergence and, therefore, greater association.

This hierarchical view proposes that patients with isolated hippocampal damage should only be impaired on those tests of memory that tap associations between the kinds of information that converge at the level of the hippocampus and not lower in the hierarchy. Such associations should involve different kinds of components because these are most likely to be represented in different neocortical regions, and are more likely to converge later. Intra-item associations and associations between similar items, likely to be represented in one neocortical region, should converge earlier in the MTL cortices. Memory representations of these associations with somewhat different properties may be formed separately in the hippocampus and MTL cortices (see O'Reilly et al., 1998). Support for this last proposal is unpublished evidence that $\mathrm{YR}$ is impaired at cued recall for both items and word pairs despite showing normal recognition for such information. O'Reilly and his colleagues propose that the hippocampus stores information in such a way as to facilitate memory pattern completion from partial cues whereas MTL cortex memory representations do not have this property.

In conclusion, three experiments have indicated that selective hippocampal damage disrupts memory for several kinds of temporal order information, but not for word pair recognition and not usually for single item recognition. This pattern of results is incompatible with the view that differently located MTL lesions disrupt memory in qualitatively the same way (as is implied by the unitary view of global amnesia) and also with the aspect of the view of Parkin and his associates, which proposes that MTL lesions disrupt temporal memory and item recognition equally. The deficit pattern of YR suggests that the impaired kinds of within-list (and possibly of between-list) temporal order memory depend on forming and retrieving cross-regional associations and that hippocampal lesions impair the storage of such associations whilst minimally affecting intra-item and within-region association 
memory formation and retrieval. It is proposed that the hippocampus is the only brain region that can rapidly form cross-regional associative memories whereas the MTL cortices are able to form the other kinds of associative memories rapidly. The implications of this suggestion for temporal order memory can be tested further by examining whether encoding temporal order information into memory, and retrieving such memories, activate several neocortical regions whereas encoding and retrieving item and within-region associations do not.

Manuscript received 8 March 1999

Revised manuscript received 6 December 1999 Revised manuscript accepted 30 March 2000

\section{REFERENCES}

Aggleton, J.P., \& Brown, M.W. (1999). Episodic memory, amnesia, and the hippocampal-anterior thalamic axis. Behavioural and Brain Science, 22, 425-489.

Aggleton, J.P., \& Shaw, C. (1996). Amnesia and recognition memory: A reanalysis of psychometric data. Neuropsychologia, 34, 51-62.

Baddeley, A., Emslie, H. \& Nimmo-Smith, I. (1994). Doors and people: A test of visual and verbal recall and recognition. Bury St. Edmunds, UK: Thames Valley Test Company.

Battig, W.F., \& Montague, W.E. (1969). Category norms for verbal items in 56 categories: A replication and extension of the Connecticut category norms. Journal of Experimental Psychology: Monograph, 80, 145.

Baxendale, S. (1997). The role of the hippocampus in recognition memory. Neuropsychologia, 35, 591-598.

Benton, A.L. (1968). Differential behavioural effects in frontal lobe disease. Neuropsychologia, 6, 53-60.

Cabeza, R., Mangels, J., Nyberg, L., Habib, R., Houle, S., McIntosh, A.R. \& Tulving, E. (1997). Brain regions differentially involved in remembering what and when: A PET study. Neuron, 19, 863-870.

Cabeza, R., \& Nyberg, L. (1997). Imaging cognition: An empirical review of PET studies with normal subjects. Journal of Cognitive Neuroscience, 9, 1-26.

Greene, R.L., Thapar, A., \& Westerman, D.L. (1998). Effects of generation on memory for order. Journal of Memory and Language, 38, 255-264.
Haist, F., Shimamura, A.P., \& Squire, L.R. (1992) On the relationship between recall and recognition memory. Journal of Experimental Psychology: Learning, Memory and Cognition, 18, 691-702.

Hanley, J.R., Davies, A.D.M., Downes, J.J., \& Mayes, A.R. (1994). Impaired recall of verbal material following rupture and repair of an anterior communicating artery aneurysm. Cognitive Neuropsychology, 11, 543-578.

Holdstock, J.S., Isaac, C.L., Cezayirli, E., Roberts, J.N., \& Mayes, A.R. (1999). What kinds of memory do selective hippocampal lesions disrupt in humans? British Neuroscience Association Abstracts, 15, 86.

Holdstock, J.S., Mayes, A.R., Cezayirli, E., Isaac, C.L., Aggleton, J.P., \& Roberts, J.N. (2000). A comparison of egocentric and allocentric spatial memory in a patient with partial selective hippocampal damage. Neuropsychologia, 38, 410-425.

Hunkin, N.M. \& Parkin, A.J. (1993). Recency judgements in Wernicke-Korsakoff and post encephalitic amnesia: Influences of proactive interference and retention interval. Cortex, 29, 485-499.

Hunkin, N.M., Parkin, A.J., \& Longmore, B.E. (1994). Aetiological variation in the amnesic syndrome: Comparisons using the list discrimination task. Neuropsychologia, 32, 819-825.

Hunkin, N.M., Stone, J.V., Isaac, C.L., Holdstock, J.S., Butterfield, R., Wallis, L.I., \& Mayes, A.R. (2000). Factor analysis of three standardised tests of memory in a clinical population. British Journal of Clinical Psychology, 39, 169-180.

Kopelman, M.D. (1989). Remote and autobiographical memory, temporal context memory and frontal atrophy in Korsakoff and Alzheimer patients. Neuropsychologia, 27, 437-460.

Kopelman, M.D., Stanhope, N., \& Kingsley, D. (1997). Temporal and spatial context memory in patients with focal frontal, temporal lobe, and diencephalic lesions. Neuropsychologia, 35, 1533-1545.

Kuçera, H. \&Francis, W.N. (1967). Computational analysis of present day American English. Providence, RI: Brown University Press.

Lezak, M.D. (1995). Neuropsychological assessment. (3rd ed.). New York: Oxford University Press.

Mangels, J.A. (1997). Strategic processing and memory for temporal order in patients with frontal lobe lesions. Neuropsychology, 11, 207-221.

Markowitsch, H.J., Weber-Luxemburger, G., Ewald, K., Kessler, J., \& Heiss, W.-D. (1997). Patients with heart attacks are not valid models for medial temporal lobe amnesia. A neuropsychological and FDG-PET

COGNITIVE NEUROPSYCHOLOGY, 2001, 18 (2) 121 
study with consequences for memory research. European Journal of Neurology, 4, 178-184.

Mayes, A.R. (1988). Human organic memory disorders. Cambridge: Cambridge University Press.

Mayes, A.R., Meudell, P.R., \& Pickering, A. (1985). Is organic amnesia caused by a selective deficit in remembering contextual information? Cortex, 21, 167-202.

Mayes, A.R., van Eijk, R., Gooding, P.A., Isaac, C.L., \& Holdstock, J.S. (1999). What are the functional deficits produced by hippocampal and perirhinal cortex lesions? Behavioural Brain Sciences, 22, 460-461.

McMackin, D., Cockburn, J., Anslow, P., \& Gaffan, D. (1995). Correlation of fornix damage with memory impairment in six cases of colloid cyst removal. Acta Neurochirugica, 135, 12-18.

Michon, J.A., \& Jackson, J.L. (1984). Attentional effort and cognitive strategies in the processing of temporal information. In J. Gibbon \& L.G. Allen (Eds.), Annals of the New York Academy of Sciences: Vol. 423. Time and time perception (pp. 298-321). New York: New York Academy of Sciences.

Milner, B. (1971). Interhemispheric differences in the localization of psychological processes in man. British Medical Bulletin, 27, 272-277.

Milner, B., Corsi, P., \& Leonard, G. (1991). Frontal lobe contribution to recency judgements. Neuropsychologia, 29, 601-618.

Mishkin, M., Vargha-Khadem, F., \& Gadian, D.G. (1998). Amnesia and the organization of the hippocampal system. Hippocampus, 8, 212-216.

Nelson, H.E. (1976). A modified card sorting test sensitive to frontal lobe deficits. Cortex, 12, 313-324.

Nelson, H.E. (1982). The National Adult Reading Test. Windsor, UK: NFER-Nelson.

O'Reilly, R.C., Norman, K.A., \& McClelland, J.L. (1998). A hippocampal model of recognition memory. In: M.I. Jordan, M.J. Kearns, and S.A. Solla (Eds.), Advances in neural information processing systems 10. Cambridge, MA: MIT Press.

Parkin, A.J. (1992). Functional significance of etiological factors in human amnesia. In L.R. Squire \& N. Butters (Eds.). Neuropsychology of memory (2nd ed.). New York: Guilford Press.

Parkin, A.J., \& Hunkin, N.M. (1993). Impaired temporal context memory on anterograde but not retrograde tests in the absence of frontal pathology. Cortex, 29, 267-280.

Parkin, A.J., \& Hunkin, N.M. (1997). How should a database on human amnesia evolve? Comments on Mayes and Downes "What do theories of the func- tional deficit(s) underlying amnesia have to explain?” Memory, 5, 99-104.

Parkin, A.J., \& Leng, N.R.C. (1993). Neuropsychology of the amnesic syndrome. Hove, UK: Lawrence Erlbaum Associates Ltd.

Parkin, A.J., Leng, N.R.C., \& Hunkin, N.M. (1990). Differential sensitivity to context in diencephalic and temporal lobe amnesia. Cortex, 26, 373-380.

Parkin, A.J., Rees, J.E., Hunkin, N.M., \& Rose, P.E. (1994). Impairment of memory following discrete thalamic infarction. Neuropsychologia, 32, 39-51.

Reed, J.M., \& Squire, L.R. (1997). Impaired recognition memory in patients with lesions limited to the hippocampal formation. Behavioural Neuroscience, 111, 667-675.

Rempel-Clower, N.L., Zola, S.M., Squire, L.R., \& Amaral, D.G. (1996). Three cases of enduring memory impairment after bilateral damage limited to the hippocampal formation. The Journal of Neuroscience, 16, 5233-5255.

Sagar, H.J., Gabrieli, J.D.E., Sullivan, E.V., \& Corkin, S. (1990). Recency and frequency discrimination in the amnesic patient H.M. Brain, 113, 581-602.

Shallice, T., \& Evans, M.E. (1978). The involvement of the frontal lobes in cognitive estimation. Cortex, 14, 294-303.

Shimamura, A.P., Janowsky, J.S., \& Squire, L.R. (1990). Memory for the temporal order of events in patients with frontal lobe lesions and amnesic patients. Neuropsychologia, 28, 803-813.

Squire, L.R. (1982). Comparisons between forms of amnesia: Some deficits are unique to Korsakoff's syndrome. Journal of Experimental Psychology: Learning, Memory and Cognition, 8, 560-571.

Squire, L.R., Knowlton, B., \& Musen, G. (1993). The structure and organization of memory. Annual Review of Psychology, 44, 453-495.

Squire, L.R., Nadel, L., \& Slater, P.C. (1981). Anterograde amnesia and memory for temporal order. Neuropsychologia, 19, 141-145.

Tzeng, O.J.L., Lee, A.T., \&Wetzel, C.D. (1979). Temporal coding in verbal information processing. Journal of Experimental Psychology: Human Learning and Memory, 5, 52-64.

Vargha-Khadem, F., Gadian, D.G., Watkins, K.E., Connelly, A., van Paesschen, W., \& Mishkin, M. (1997). Differential effects of early hippocampal pathology on episodic and semantic memory. Science, 277, 376-380.

Victor, M., Adams, R.D., \& Collins, G.H. (1989). The Wernicke-Korsakoff Syndrome and related neurologic 
disorders due to alcoholism and malnutrition, (2nd ed.). Philadelphia, PA: Davis.

Warrington, E.K. (1984). Recognition Memory Test. Windsor, UK: NFER-Nelson.

Wechsler, D. (1945). A standardized memory scale for clinical use. Journal of Psychology, 19, 87-95.

Wechsler, D. (1987). Wechsler Memory Scale-Revised. New York: Psychological Corporation.

Wechsler, D. (1955). Wechsler Adult Intelligence Scale manual. New York: Psychological Corporation.

Wechsler, D. (1981). Wechsler Adult Intelligence ScaleRevised. San Antonio, TX: Psychological Corporation.

Yonelinas, A.P. (1997). Recognition memory ROCs for item and associative information: the contribution of recollection and familiarity. Memory and Cognition, 25, 747-763.

Yonelinas, A.P., Kroll, N.E.A., Dobbins, I., Lazzara, M., \& Knight, R.T. (1998). Recollection and familiarity deficits in amnesia: Convergence of rememberknow, process dissociation, and receiver operating characteristic data. Neuropsychology, 12, 323-339.

Zacks, R.T., Hasher, L., Alba, J.W., Sanft, H., \& Rose, K.C. (1984). Is temporal order encoded automatically? Memory and Cognition, 12, 387-394.

Zola-Morgan, S., \& Squire, L.R. (1993). Neuroanatomy of memory. Annual Review of Neuroscience, 16, 547563. 